\title{
Normalized Robust FOPID Controller Regulation Based on Small Gain Theorem
}

\author{
Shuo Zhang $\mathbb{D D}^{1}$ and Lu Liu $\mathbb{D}^{2}$ \\ ${ }^{1}$ Department of Applied Mathematics, Northwestern Polytechnical University, Xi'an 710072, China \\ ${ }^{2}$ School of Marine Science and Technology, Northwestern Polytechnical University, Xi'an 710072, China \\ Correspondence should be addressed to Lu Liu; liulu12201220@nwpu.edu.cn
}

Received 6 April 2018; Accepted 9 August 2018; Published 27 September 2018

Academic Editor: Lucia Valentina Gambuzza

Copyright (c) 2018 Shuo Zhang and Lu Liu. This is an open access article distributed under the Creative Commons Attribution License, which permits unrestricted use, distribution, and reproduction in any medium, provided the original work is properly cited.

\begin{abstract}
In this paper, a normalized robust FOPID controller regulation algorithm is proposed. Only one parameter $k$ is necessary to be tuned in the controller regulation process, so the proposed control algorithm is convenient to be applied on both fractional-order systems and integer-order systems. A robustness evaluation function is constructed based on the small gain theorem. Larger robustness evaluation function value will help the system achieve better robustness performance. Another parameter, $\beta$, is also available to serve as a tuning knob when larger robust evaluation function value is needed. Therefore, the controlled systems can be stabilized and can achieve quite satisfactory robust control performance using the proposed algorithm. The corresponding robust analysis results are obtained according to different conditions in the discussion. For a special case of widely used fractional-order systems, the FOPI and FOID controllers are presented based on the same tuning scheme together with their robustness discussion. Some examples are shown to verify the robustness of systems controlled by the proposed algorithm.
\end{abstract}

\section{Introduction}

In 1695, fractional calculus was first mentioned in Leibniz's letter to L'Hospital, as a generalization of classical integerorder calculus. Due to the lack of application background, fractional calculus was regarded as a pure mathematical issue for a long time. However, recently, more and more researchers have focused on fractional calculus-related topics and discovered its applications in numerous fields, such as physics [1], biology [2], engineering [3-5], and neuroscience $[6,7]$. In particular, fractional-order modelling and fractional-order control technology have achieved a rapid development [8-12]. A number of physical processes are proven to be better expressed by fractional-order models [1, 13]. In other words, fractional calculus could provide a more precise description for modelling. References $[4,14-16]$ point out that fractional calculus could provide one more dimension in control process. Therefore, it owns more potential in enhancing system robustness as well as transient control performance.
Fractional-order PID (FOPID or $\mathrm{PI}^{\lambda} \mathrm{D}^{\mu}$ ) control is an important application of fractional calculus which was presented in [5]. Different from classical PID controllers, FOPID controllers have two more parameters, namely, integral order $\lambda$ and differential order $\mu$. When $\lambda=\mu=1$, the FOPID controller equals to the classical PID controller. Thus, PID controller is a special case of FOPID controller which has wider application domains. To achieve the controller design specifications of the added two parameters $\lambda$ and $\mu$, Petras [14] proposed a digital FOPID controller with hardware implementation for a DC motor. For the given digital controller, the author realized the digital implementation and analogue realization by microprocessors and fractance circuits, respectively. Reference [17] proposed a frequency domain approach of tuning FOPID controller. The tuning and auto-tuning method of FOPID controller for industry applications was studied in [18]. The proposed controller was tuned to satisfy five different design specifications. A FOPID controller used for the stabilization of fractionalorder time delay systems was designed in [19]. The method 
can be used to guarantee different gain and phase margin specifications. An optimal FOPID controller was proposed by Zamani et al. based on the minimum integral squared error (ISE) criterion to meet specified gain and phase margins requirements. Some FOPID tuning methods based on flat phase property for servo control and disturbance compensation for different motion systems were demonstrated in [9]. Reference [20] presented a FOPID design algorithm for an automatic voltage regulator using particle swarm optimization. Many related studies show that the control performance under FOPID controller outperforms the other kinds of controllers. Moreover, Xue [21] provides some efficient numerical implementations of FOPID controller with open-source MATLAB codes.

For control issues, robustness analysis is quite important because it relates to system stability with respect to internal and external disturbances. For classical PID controller, its robustness studies have obtained a lot of attention. Comparatively, the studies on robustness of fractional-order systems are quite limited. In our paper, we investigate a normalized regulation algorithm for robust FOPID controller design. Different from other controller tuning methods, only one parameter $k$ is necessary to be tuned. Therefore, the proposed controller is convenient and simple to be applied on different kinds of systems, including fractional-order and integer-order ones. The controlled system robustness is analyzed by discussing a robust evaluation function based on the small gain theorem. Larger robust evaluation function could bring about better robust performance. Moreover, another parameter, $\beta$, can also serve as a tuning knob to help get large enough robust evaluation function. The robustness of a special kind of fractional-order systems which is commonly used in practice is also considered under FOPI and FOID controllers.

The rest of paper is organised as follows. Section 2 introduces the preliminaries, including fractional integral and derivative, and fractional-order systems described in time and frequency domains. Robust analysis of the proposed FOPID controllers is listed in Section 3. Two control parameters $k$ and $\beta$ are discussed in two subsections, respectively. Section 4 analyzes the robustness for a special case of the fractional-order system under FOPI and FOID controllers. The simulation results which show the effectiveness and practicability of the proposed control algorithm are demonstrated in Section 5. At last, conclusion is drawn in Section 6.

\section{Preliminaries}

2.1. Fractional Integral and Derivative. In the studies on fractional integral and derivative, there are three definitions which are widely used, namely, Grunwald-Letnikov definition, Riemann-Liouville definition, and Caputo definition [5]. These three definitions own different properties, such that they are applied in different fields of engineering and computing science. Due to the discreteness of the Grunwald-Letnikov definition, it is always utilized in computing and simulation. Thus, we mainly introduce Riemann-Liouville definition and Caputo definition here.
Definition 1. For an integrable function $f(t)$, its RiemannLiouville integral of order $q>0$ is defined as

$$
{ }_{0} J_{t}^{q} f(t)=\frac{1}{\Gamma(q)} \int_{0}^{t}(t-\tau)^{q-1} f(\tau) d \tau .
$$

The Laplace transform of the Riemann-Liouville integral is

$$
L\left\{{ }_{0} J_{t}^{q} f(t) ; s\right\}=s^{-q} F(s),
$$

where $q>0, L\{\cdot\}$ denotes the Laplace transform, $s$ is the variable operator in Laplace transform, and $F(s)$ is the Laplace transform of $f(t)$.

Definition 2. For a function $f(t) \in C^{n+1}([0,+\infty], R)$, its Riemann-Liouville derivative of order $\alpha$ is described by

$$
{ }_{0}^{L} D_{t}^{\alpha} f(t)=\frac{1}{\Gamma(n-\alpha)}\left(\frac{d}{d t}\right)^{n} \int_{t_{0}}^{t} \frac{f(\tau)}{(t-\tau)^{\alpha+1-n}} d \tau,
$$

where the positive integer $n$ satisfies $n-1<\alpha \leq n$.

The Laplace transform of the Riemann-Liouville derivative is

$$
L\left\{{ }_{0}^{L} D_{t}^{\alpha} f(t) ; s\right\}=s^{\alpha} F(s)-\sum_{k=0}^{n-1} s^{k}\left[{ }_{0}^{L} D_{t}^{\alpha-k-1} f(t)\right]_{t=0},
$$

where $n-1<\alpha \leq n$.

Caputo operator only owns derivative definition, which is given as follows.

Definition 3. For a function $f(t) \in C^{n+1}([0,+\infty], R)$, its Caputo derivative of order $\alpha$ is defined as

$$
{ }_{0}^{C} D_{t}^{\alpha} f(t)=\frac{1}{\Gamma(n-\alpha)} \int_{t_{0}}^{t} \frac{f^{(n)}(\tau)}{(t-\tau)^{\alpha+1-n}} d \tau,
$$

where the positive integer $n$ satisfies $n-1<\alpha \leq n$.

The Laplace transform of the Caputo derivative is

$$
L\left\{{ }_{0}^{C} D_{t}^{\alpha} f(t) ; s\right\}=s^{\alpha} F(s)-\sum_{k=0}^{n-1} s^{\alpha-k-1} f^{(k)}(0),
$$

where $n-1<\alpha \leq n$.

2.2. Fractional-Order Systems Described in Time and Frequency Domains. In the time domain, fractional-order systems can be described by fractional-order derivative equations, such as the following common one, i.e.,

$$
a_{10}^{L, C} D_{t}^{\alpha} y(t)+a_{20}^{L, C} D_{t}^{\beta} y(t)+1=b u(t),
$$

where $u(t)$ and $y(t)$ denote the input and output of the fractional-order system, respectively. 
It should be noted that Riemann-Liouville and Caputo derivatives have no difference when their initial conditions are null. Without regard for the initial conditions of fractional derivative operators, the above fractional-order systems can be described by the following transfer function in the frequency domain:

$$
\frac{Y(s)}{U(s)}=\frac{b}{a_{1} s^{\alpha}+a_{2} s^{\beta}+1},
$$

where $Y(s)$ and $U(s)$ are the Laplace transform of $y(t)$ and $u(t)$, respectively. The above system can be used to describe both fractional-order or integer-order systems, for example, the typical second-order system with $\alpha=2, \beta=1$. Moreover, a lot of high-order systems can be approximated by this system [21]. Therefore, it is worth to be further studied. In the next section, we will consider the above fractional-order system in frequency domain.

\section{Robust Analysis for Fractional-Order Systems under FOPID Controllers}

Consider a fractional-order transfer function described by

$$
P(s)=\frac{b}{a_{1} s^{\alpha}+a_{2} s^{\beta}+1},
$$

where $b>0$ is the DC gain, $a_{1}$ and $a_{2}$ are the positive constant coefficients, and the fractional orders satisfy $0<\beta<\alpha<2$.

Next, we give the corresponding FOPID controller designed as

$$
C(s)=k_{p}+\frac{k_{i}}{s^{\lambda}}+k_{d} s^{\mu}
$$

where fractional orders are chosen as $\lambda=\beta$ and $\mu=\alpha-\beta$ and the controller parameters are set as $k_{p}=k a_{2}, k_{i}=k$, and $k_{d}=k a_{1}$. The constant $k>0$ can be designed according to the practical control process.

Based on systems (1) and (2), the normalized open-loop transfer function $L(s)=P(s) C(s)$ is obtained as

$$
L(s)=\frac{b k}{s^{\beta}} .
$$

Besides, the gain crossover frequency $\omega_{c}$ of the open-loop transfer function $L(s)$ is gained by

$$
\omega_{\mathrm{c}}=(b k)^{1 / \beta}
$$

The closed-loop transfer function $L_{\mathrm{c}}(s)=(L(s)) /(1+L(s))$ can be achieved as

$$
L_{\mathrm{c}}(s)=\frac{1}{\left(s^{\beta} / b k\right)+1}
$$

Due to $0<\beta<2$ and $b, k>0$, it is straightforward to obtain the stability of $L_{\mathrm{c}}(s)$ based on the previous results in $[22,23]$.

Then, we analyze the robustness of the proposed FOPID controller (2) by employing the small gain theorem, which has been widely used in FOPID control [24-27]. According to small gain theorem, the robust stability condition can be represented by

$$
\delta_{p}(\omega)<V(\omega, \beta, k) \triangleq \frac{|1+P(\omega i) C(\omega i)|}{|P(\omega i) C(\omega i)|}, \quad \forall \omega>0,
$$

where $\delta_{p}(\omega)$ denotes the multiplicative norm-bound uncertainty of transfer function $P(s)$ in (9). $V(\omega, \beta, k)$ is the robust evaluation function, and larger $V(\omega, \beta, k)$ means that bigger modelling uncertainty could be tolerated without breaking the robust stability of the controlled system.

Due to systems (1), (2), and (5), we gain the $V(\omega, \beta, k)$ as

$$
\begin{aligned}
V(\omega, \beta, k) & =\frac{\left|1+\left((b k) /\left((\omega i)^{\beta}\right)\right)\right|}{\left|(b k) /\left((\omega i)^{\beta}\right)\right|}=\frac{\left|1+\left((b k) /\left(\omega^{\beta}\right)\right) i^{-\beta}\right|}{(b k) /\left(\omega^{\beta}\right)} \\
& =\left(\frac{\omega^{2 \beta}}{b^{2} k^{2}}+\frac{2 \omega^{\beta}}{b k} \cos \left(\frac{\beta \pi}{2}\right)+1\right)^{1 / 2} .
\end{aligned}
$$

Calculating partial derivatives of $V(\omega, \beta, k)$ with respect to $k$ and $\beta$, respectively, it has

$$
\begin{aligned}
& \frac{\partial V(\omega, \beta, k)}{\partial k}=-\frac{\left(\omega^{2 \beta} /\left(b^{2} k^{3}\right)\right)+\left(\omega^{\beta} /\left(b k^{2}\right)\right) \cos ((\beta \pi) / 2)}{\sqrt{\left(\left(\omega^{2 \beta} /\left(b^{2} k^{2}\right)\right)+\left(\left(2 \omega^{\beta}\right) /(b k)\right) \cos ((\beta \pi) / 2)+1\right)}}, \\
& \frac{\partial V(\omega, \beta, k)}{\partial \beta}=\frac{\left(\omega^{\beta} /\left(b k^{2}\right)\right)\left[\left(\left(\omega^{\beta} /\left(b k^{2}\right)\right)+\cos ((\beta \pi) / 2)\right) \ln \omega-(\pi / 2) \sin ((\beta \pi) / 2)\right]}{\sqrt{\left(\left(\omega^{2 \beta} /\left(b^{2} k^{2}\right)\right)+\left(2^{\omega \beta} / b k\right) \cos ((\beta \pi) / 2)+1\right)}} .
\end{aligned}
$$


Next, we give the robust analysis by discussing the control parameters $k$ and $\beta$, respectively.

3.1. Robust Analysis for Control Parameter $k$. With regard to the robustness of the designed FOPID controller in (10), we only analyze the control parameter $k>0$ under some fixed $0<\beta_{*}<2$ and $\omega_{*}>0$. In detail, we give two cases according to the value of the $\beta_{*}$.

Case 1. $0<\beta_{*} \leq 1$.

When $0<\beta_{*} \leq 1$, it is obvious that

$$
\frac{\partial V\left(\omega_{*}, \beta_{*}, k\right)}{\partial k}<0, \quad \text { for } k>0,0<\beta_{*} \leq 1, \omega_{*}>0 .
$$

That means smaller $k>0$ could bring better robust performance for any fixed $0<\beta_{*} \leq 1$ and $\omega_{*}>0$. Besides, it should be noted that the gain crossover frequency $\omega_{c}$ of the open-loop transfer function $L(s)$ depends on the value of $k$ based on (12). Thus, control parameter $k$ has to be chosen to keep $\omega_{c}$ in a suitable range firstly. Then, $k>0$ should be a small enough constant to obtain better robustness.

When $\beta_{*}=0.5, \omega_{*}=5$, and $b=1$, we give the diagram between $V\left(\omega_{*}, \beta_{*}, k\right)$ and $k$ in Figure 1, which shows that $V\left(\omega_{*}, \beta_{*}, k\right)$ is monotonously decreasing with respect to $k$.

Case 2. $1<\beta_{*}<2$.

For fixed $1<\beta_{*}<2$ and $\omega_{*}>0$, there exists a unique solution.

$$
\bar{k}=-\frac{\omega_{*}^{\beta_{*}}}{b \cos \left(\left(\beta_{*} \pi\right) / 2\right)},
$$

such that

$$
\frac{\partial V\left(\omega_{*}, \beta_{*}, k\right)}{\partial k}=0 .
$$

In addition, it has

$$
\frac{\partial V\left(\omega_{*}, \beta_{*}, k\right)}{\partial k}<0, \quad \text { for } 0<k<\bar{k}, 1<\beta_{*}<2, \omega_{*}>0,
$$

$$
\frac{\partial V\left(\omega_{*}, \beta_{*}, k\right)}{\partial k}>0, \quad \text { for } k>\bar{k}, 1<\beta_{*}<2, \omega_{*}>0 .
$$

Thus, $\partial V\left(\omega_{*}, \beta_{*}, k\right) / \partial k$ is monotonously decreasing at $0<k<\bar{k}$ and monotonously increasing at $k>\bar{k}$. It owns a minimum value at $k=\bar{k}$, which means that the robustness of the designed FOPID controller is worst at $k=\bar{k}$. Note that

$$
V\left(\omega_{*}, \beta_{*}, \frac{\bar{k}}{2}\right)=V\left(\omega_{*}, \beta_{*},+\infty\right)=1 .
$$

So it has

$$
V\left(\omega_{*}, \beta_{*}, k_{1}\right)>V\left(\omega_{*}, \beta_{*}, k_{2}\right),
$$

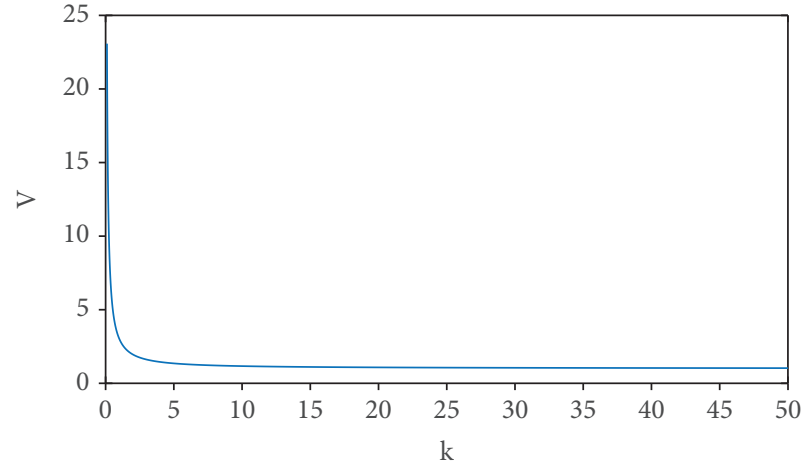

FIgURE 1: Diagram between $V\left(\omega_{*}, \beta_{*}, k\right)$ and $k$ under $\beta_{*}=0.5$.

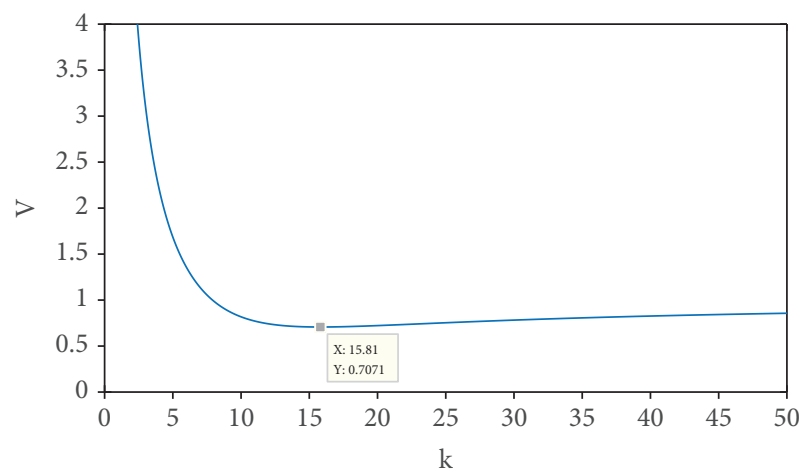

FIgURE 2: Diagram between $V\left(\omega_{*}, \beta_{*}, k\right)$ and $k$ under $\beta_{*}=1.5$.

for any $0<k_{1}<\bar{k} / 2$ and $k_{2}>\bar{k} / 2$. Thus, for any fixed $1<\beta_{*}<2$ and $\omega_{*}>0$, small enough $k>0$ does well in the robust performance of the controlled system.

Due to the relationship in (12) between control parameter $k$ and gain crossover frequency $\omega_{c}, k$ may be designed in a limited interval. Assume that $k_{\min }$ and $k_{\max }$ are the minimum and maximum for the available control parameter $k$, respectively, then $k$ could be chosen as

$$
k=\underset{x}{\arg \max } V\left(\omega_{*}, \beta_{*}, x\right), \quad x \in\left\{k_{\min }, k_{\max }\right\},
$$

to realize the best robust performance.

When $\beta_{*}=1.5, \omega_{*}=5$, and $b=1, V\left(\omega_{*}, \beta_{*}, k\right)$ has a minimum value at

$$
\bar{k}=-\frac{\omega_{*}^{\beta_{*}}}{b \cos \left(\left(\beta_{*} \pi\right) / 2\right)} \approx 15.81 .
$$

We give the diagram between $V\left(\omega_{*}, \beta_{*}, k\right)$ and $k$ in Figure 2, which verifies the obtained results.

3.2. Robust Analysis for Control Parameter. In this subsection, another control parameter $\beta$ is discussed to analyze the robustness of the proposed FOPID controller under some fixed $k_{*}>0$ and $\omega_{*}>0$. According to (7), we analyze the monotonicity of $\left(\omega_{*}^{\beta} \ln \omega_{*}\right) /\left(b k_{*}^{2}\right), \cos ((\beta \pi) / 2) \ln \omega_{*}$, and $\sin ((\beta \pi) / 2)$ and list the following cases. 
Case 3. $0<\omega_{*}<1$.

If $0<\omega_{*}<1$ and $\left(\omega_{*}^{2} /\left(b k_{*}^{2}\right)\right) \geq 1$, it satisfies

$$
\frac{\partial V\left(\omega_{*}, \beta_{*}, k_{*}\right)}{\partial k}<0, \quad \text { for } 0<\beta>2, k_{*}>0,<\omega_{*}<1
$$

So $V\left(\omega_{*}, \beta, k_{*}\right)$ is monotonously decreasing at $0<\beta<2$. Thus, to obtain better robustness, control parameter $\beta$ should be chosen small enough.

When $\omega_{*}=0.5, k=0.2$, and $b=1\left(\omega_{*}^{2} /\left(b k_{*}^{2}\right)=6.25>1\right)$, the diagram between $V\left(\omega_{*}, \beta_{*}, k\right)$ and $\beta$ is shown in Figure 3 , which verifies that $V\left(\omega_{*}, \beta_{*}, k\right)$ is monotonously decreasing with respect to $\beta$.

If $0<\omega_{*}<1$ and $\omega_{*}^{2} /\left(b k_{*}^{2}\right)<1$, there exists a unique solution $\bar{\beta}$ for the equation:

$$
\left(\frac{\omega_{*}^{\beta}}{b k_{*}^{2}}+\cos \left(\frac{\beta \pi}{2}\right)\right) \ln \omega_{*}-\frac{\pi}{2} \sin \left(\frac{\beta \pi}{2}\right)=0,
$$

which is also the unique solution of

$$
\frac{\partial V\left(\omega_{*}, \beta, k_{*}\right)}{\partial \beta}=0 .
$$

Moreover, it satisfies

$\frac{\partial V\left(\omega_{*}, \beta, k_{*}\right)}{\partial \beta}<0, \quad$ for $0<\beta<\bar{\beta}, k_{*}>0,0<\omega_{*}<1$,

$\frac{\partial V\left(\omega_{*}, \beta, k_{*}\right)}{\partial \beta}>0, \quad$ for $\bar{\beta}<\beta<2, k_{*}>0,0<\omega_{*}<1$

That means $V\left(\omega_{*}, \beta, k_{*}\right)$ is monotonously decreasing at $0<\beta<\bar{\beta}$ and monotonously increasing at $\bar{\beta}<\beta<2$. Due to

$$
\begin{aligned}
V\left(\omega_{*}, 0, k_{*}\right) & =\left(\frac{1}{b^{2} k^{2}}+\frac{2}{b k}+1\right)^{1 / 2}>\left(\frac{\omega^{4}}{b^{2} k^{2}}-\frac{2 \omega^{2}}{b k}+1\right)^{1 / 2} \\
& =V\left(\omega_{*}, 2, k_{*}\right)
\end{aligned}
$$

control parameter $\beta>0$ should be designed as a small enough order, which can obtain large enough $V\left(\omega_{*}, \beta, k_{*}\right)$ to improve the robust performance.

When $\omega_{*}=0.5, k=1$, and $b=1\left(\omega_{*}^{2} /\left(b k_{*}^{2}\right)=0.25<1\right)$, the diagram between $V\left(\omega_{*}, \beta_{*}, k\right)$ and $\beta$ is shown in Figure 4 , which gives $\bar{\beta} \approx 1.81$.

Remark 1. When $\omega_{*}=1$, it has

$$
\frac{\partial V\left(\omega_{*}, \beta, k_{*}\right)}{\partial \beta}<0, \quad \text { for } 0<\beta<2, k_{*}>0, \omega_{*}=1 .
$$

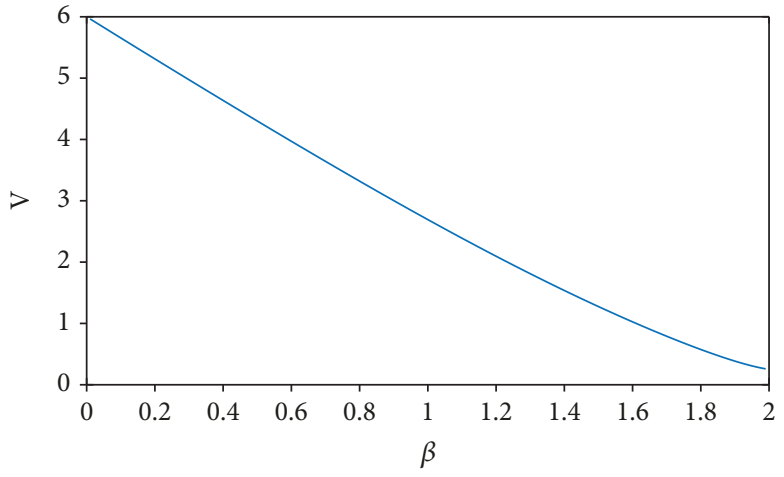

Figure 3: Diagram between $V\left(\omega_{*}, \beta_{*}, k\right)$ and $\beta$ under $\omega_{*}=0.5$ and $\omega_{*}^{2} /\left(b k_{*}^{2}\right)=6.25$.

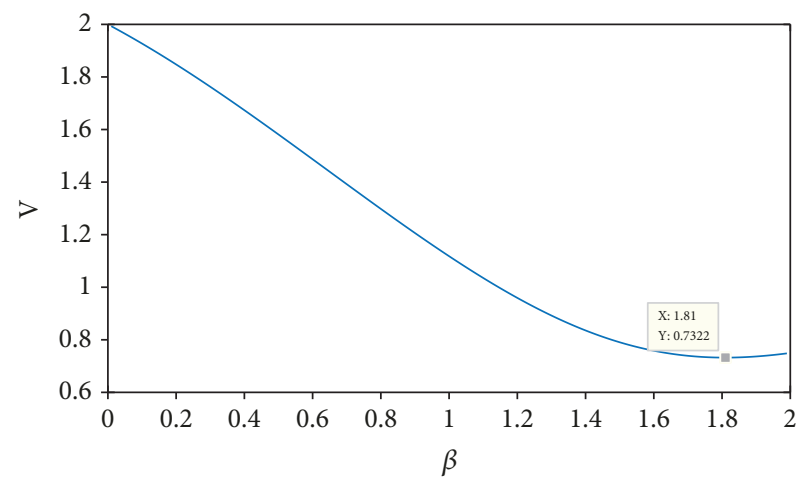

FIgURE 4: Diagram between $V\left(\omega_{*}, \beta_{*}, k\right)$ and $\beta$ under $\omega_{*}=0.5$ and $\omega_{*}^{2} /\left(b k_{*}^{2}\right)=0.25$.

Thus, $V\left(\omega_{*}, \beta, k_{*}\right)$ is monotonously decreasing at $0<\beta<2$. The robust analysis result is similar with that in Case 3 $\left(0<\omega_{*}<1\right.$ and $\left.\omega_{*}^{2} /\left(b k_{*}^{2}\right) \geq 1\right)$.

Case 4. $\omega_{*}>1$.

If $\omega_{*}>1$ and $\left(\omega_{*} \ln \omega_{*}\right) /\left(b k_{*}^{2}\right) \geq \pi / 2$, it gives

$$
\frac{\partial V\left(\omega_{*}, \beta, k_{*}\right)}{\partial \beta}>0, \quad \text { for } 0<\beta<2, k_{*}>0, \omega_{*}>1 .
$$

In other words, $V\left(\omega_{*}, \beta, k_{*}\right)$ is monotonously increasing at $0<\beta<2$. So $\beta$ should be chosen as a big enough one to obtain better robust performance.

When $\omega_{*}=3, k=1$, and $b=1 \quad\left(\left(\omega_{*} \ln \omega_{*}\right) /\left(b k_{*}^{2}\right) \approx\right.$ $3.3>\pi / 2)$, the diagram between $V\left(\omega_{*}, \beta_{*}, k\right)$ and $\beta$ is shown in Figure 5, which verifies that $V\left(\omega_{*}, \beta_{*}, k\right)$ is monotonously increasing with respect to $\beta$.

If $\omega_{*}>1,\left(\omega_{*} \ln \omega_{*}\right) /\left(b k_{*}^{2}\right)<\pi / 2$ and $\omega_{*}^{2} /\left(b k_{*}^{2}\right)<1$, there exists a unique solution $0<\bar{\beta}<1$ for

$$
\frac{\partial V\left(\omega_{*}, \beta, k_{*}\right)}{\partial \beta}=0
$$




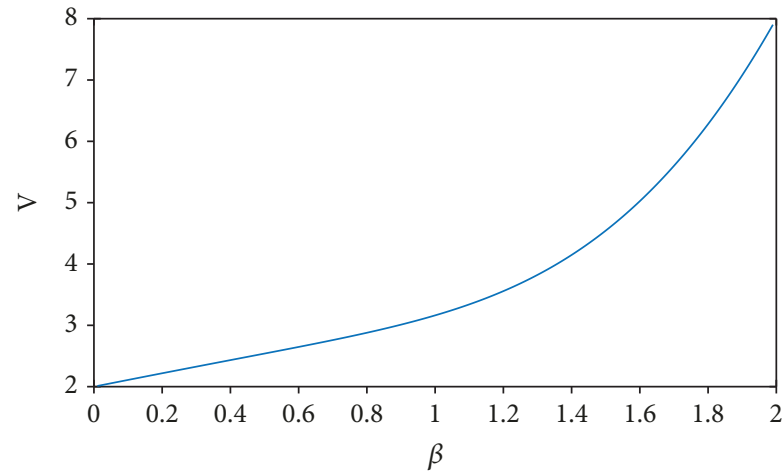

FIGURE 5: Diagram between $V\left(\omega_{*}, \beta_{*}, \mathrm{k}\right)$ and $\beta$ under $\omega_{*}=3$ and $\left(\omega_{*} \ln \omega_{*}\right) /\left(b k_{*}^{2}\right) \approx 3.3$.

Then, it has

$\frac{\partial V\left(\omega_{*}, \beta, k_{*}\right)}{\partial \beta}>0, \quad$ for $0<\beta<\bar{\beta}, k_{*}>0, \omega_{*}>1$,

$$
\frac{\partial V\left(\omega_{*}, \beta, k_{*}\right)}{\partial \beta}<0, \quad \text { for } \bar{\beta}<\beta<2, k_{*}>0, \omega_{*}>1 .
$$

It implies that $V\left(\omega_{*}, \beta, k_{*}\right)$ is monotonously increasing at $0<\beta<\bar{\beta}$ and monotonously decreasing at $\bar{\beta}<\beta<2$. So $\beta$ should be chosen as $\beta=\bar{\beta}$ to achieve the maximum value of $V\left(\omega_{*}, \beta, k_{*}\right)$.

When $\omega_{*}=1.4, k=2$, and $b=1 \quad\left(\left(\omega_{*} \ln \omega_{*}\right) /\left(b k_{*}^{2}\right) \approx\right.$ $0.12<\pi / 2$ and $\left.\omega_{*}^{2} / b k_{*}^{2} \approx 0.49<1\right)$, the diagram between $V\left(\omega_{*}, \beta_{*}, k\right)$ and $\beta$ is shown in Figure 6 , which gives $\bar{\beta} \approx 0.21$.

If $\omega_{*}>1,\left(\omega_{*} \ln \omega_{*}\right) /\left(b k_{*}^{2}\right)<\pi / 2$, and $\omega_{*}^{2} /\left(b k_{*}^{2}\right)>1$, there exists two solutions $0<\bar{\beta}_{1}<1$ and $1<\bar{\beta}_{2}<2$ for

$$
\frac{\partial V\left(\omega_{*}, \beta, k_{*}\right)}{\partial \beta}=0
$$

The other values of partial derivative are listed as

$$
\begin{aligned}
& \frac{\partial V\left(\omega_{*}, \beta, k_{*}\right)}{\partial \beta}>0, \quad \text { for } 0<\beta<\bar{\beta}_{1}, k_{*}>0, \omega_{*}>1, \\
& \frac{\partial V\left(\omega_{*}, \beta, k_{*}\right)}{\partial \beta}<0, \quad \text { for } \bar{\beta}_{1}<\beta<\bar{\beta}_{2}, k_{*}>0, \omega_{*}>1,
\end{aligned}
$$

$$
\frac{\partial V\left(\omega_{*}, \beta, k_{*}\right)}{\partial \beta}>0, \quad \text { for } \bar{\beta}_{2}<\beta<2, k_{*}>0, \omega_{*}>1
$$

It means that $V\left(\omega_{*}, \beta, k_{*}\right)$ is monotonously increasing at $0<\beta<\bar{\beta}_{1}$, monotonously decreasing at $\bar{\beta}_{1}<\beta<\bar{\beta}_{2}$, and

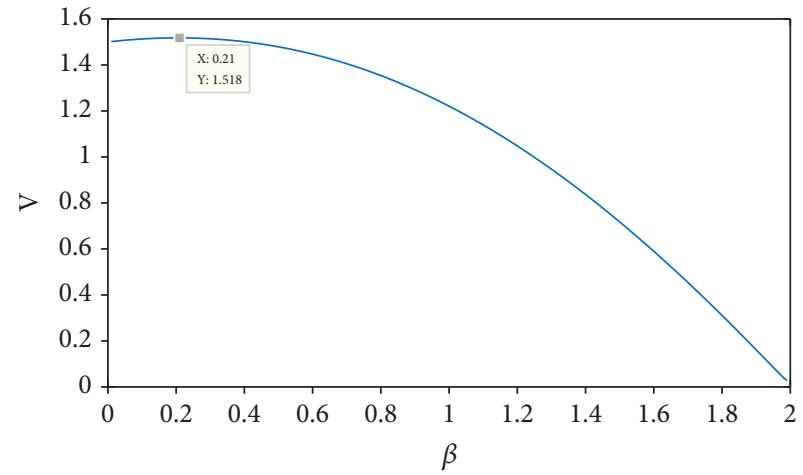

FIgURE 6: Diagram between $V\left(\omega_{*}, \beta_{*}, k\right)$ and $\beta$ under $\omega_{*}=1.4$, $\left(\omega_{*} \ln \omega_{*}\right) /\left(b k_{*}^{2}\right) \approx 0.12$, and $\omega_{*}^{2} /\left(b k_{*}^{2}\right) \approx 0.49$.

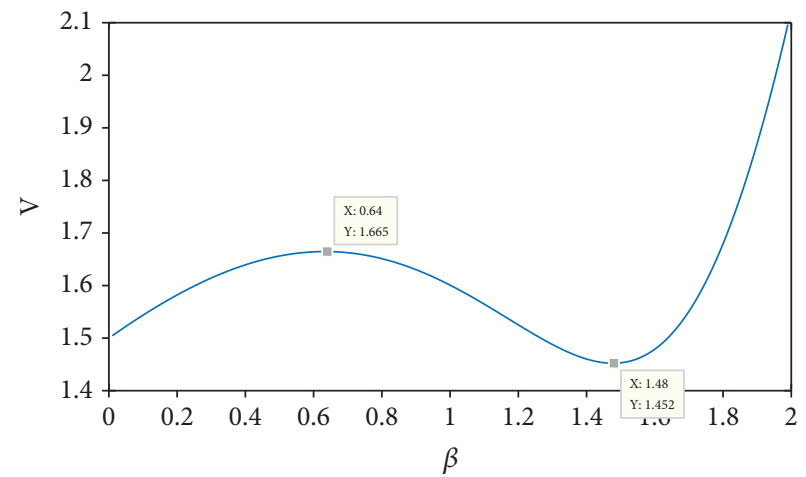

FIgURE 7: Diagram between $V\left(\omega_{*}, \beta_{*}, k\right)$ and $\beta$ under $\omega_{*}=2.5$, $\left(\omega_{*} \ln \omega_{*}\right) /\left(b k_{*}^{2}\right) \approx 0.57$, and $\omega_{*}^{2} /\left(b k_{*}^{2}\right) \approx 1.56$.

monotonously increasing at $\bar{\beta}_{2}<\beta<2$. As a conclusion, $\beta$ ought to be designed as

$$
\beta=\underset{x}{\arg \max } V\left(\omega_{*}, x, k_{*}\right), \quad x \in\left\{\bar{\beta}_{1}, \beta_{3}\right\},
$$

where $\beta_{3} \longrightarrow 2^{-}$

When $\omega_{*}=2.5, k=2$, and $b=1\left(\left(\omega_{*} \ln \omega_{*}\right) /\left(b k_{*}^{2}\right) \approx\right.$ $0.57<\pi / 2$ and $\left.\omega_{*}^{2} /\left(b k_{*}^{2}\right) \approx 1.56>1\right)$, the diagram between $V\left(\omega_{*}, \beta_{*}, k\right)$ and $\beta$ is shown in Figure 7 , which gives $\bar{\beta}_{1} \approx 0.64$ and $\bar{\beta}_{2} \approx 1.48$.

Remark 2. The control parameter $\beta$ comes from the original fractional-order transfer function in (9). However, due to the unavoidable internal or external disturbances, the real $\beta$ in fractional-order transfer function (1) may be estimated inaccurately. Thus, control parameter $\beta$ in FOPID controller is always chosen in a neighborhood of $\beta$ in fractional-order system (1). The above subsection is the global robust analysis for control parameter $0<\beta<2$. For a given fractional-order system (1), $\beta$ should be considered locally. When $a_{2}=0$, some special cases for the corresponding FOPID controllers are proposed in the next section, where the control parameter $\beta$ will be discussed globally in $\beta \in(0,2)$. 


\section{Robust Analysis for Some Special Cases}

For fractional-order system in (9), the control parameter $\beta$ is important in the FOPID controller. In this section, we give a special case of fractional-order system (1) with $a_{2}=0$, i.e.,

$$
P(s)=\frac{b}{a_{1} s^{\alpha}+1},
$$

where $b>0, a_{1}>0$, and $0<\alpha<2$. When $\alpha=1$, system (8) is the most widely used first-order system in practical application. It can also be applied to approximate different kinds of high-order systems [21].

Based on the FOPID controller in (10), $\beta$ could be designed as any value in $\beta \in(0, \alpha]$. Thus, we give two FO controllers in the following.

4.1. FOPI Controller. For fractional-order system in (42), we design the corresponding FOPI controller described by

$$
C(s)=k_{p}+\frac{k_{i}}{s^{\lambda}},
$$

where fractional order is chosen as $\lambda=\alpha$ and the controller parameters are set as $k_{p}=k a_{1}, k_{i}=k$. The constant $k>0$ is similar with controller (2). Then, the open-loop transfer function $L(s)=P(s) C(s)$ can be obtained as

$$
L(s)=\frac{b k}{s^{\alpha}},
$$

which is similar to that of (11). Thus, the robust analysis for control parameters $k$ and $\alpha$ could refer to the above results in the last section.

4.2. FOID Controller. We give the corresponding FOID controller for fractional-order system (8) which is designed as

$$
C(s)=\frac{k_{i}}{s^{\lambda}}+k_{d} s^{\mu},
$$

where fractional order is chosen as $\mu=\alpha-\lambda$ and the controller parameters are set as $k_{i}=k, k_{d}=k a_{1} . k>0$ and $0<\lambda<\alpha$ are the control parameters to be determined. Under the controller (10), the open-loop transfer function $L(s)=P(s) C(s)$ can be obtained as

$$
L(s)=\frac{b k}{s^{\lambda}},
$$

which is similar to that of (11).

Note that $\lambda$ in controller (10) can be chosen in an interval $0<\lambda<\alpha$. Thus, its robustness can be analyzed globally referring to Remark 2 . The corresponding robust analysis could follow the obtained result in Section 3.

\section{Robustness Analysis Examples}

In this section, two controlled plants with the studied formulation in (9) and (42) are used to verify the effectiveness of the

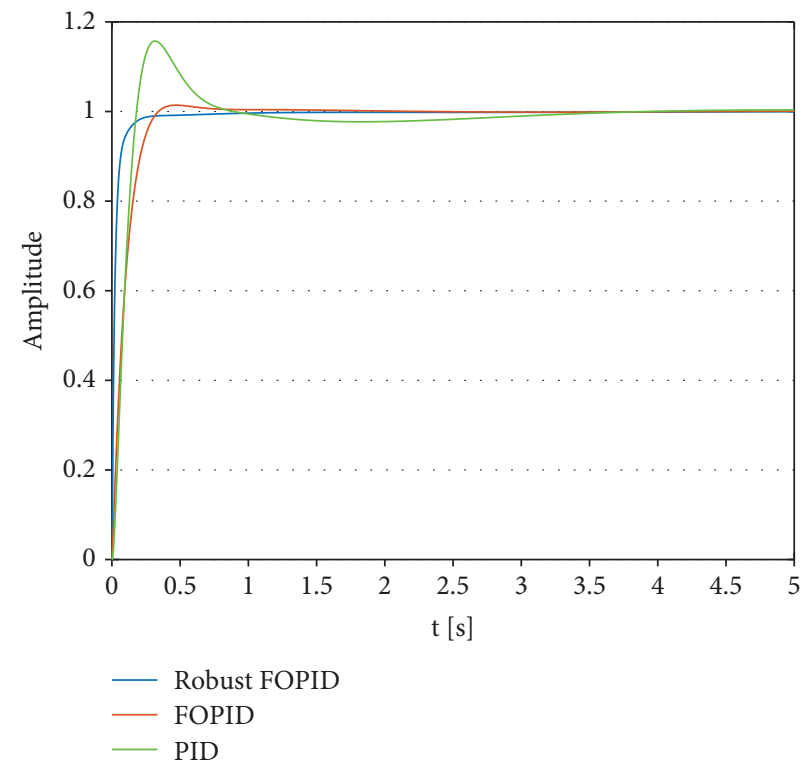

FIgURE 8: Step response comparison of $G_{1}(s)$.

proposed controller. The first system $G_{1}(s)$ is in the form of (9) with $a_{1}=0.8, a_{2}=0.5, \alpha=2.2, \beta=0.9$, and $b=1$, and the other system $G_{2}(s)$ is in the form of (42) with $a_{1}=1$, $\alpha=1.5$, and $b=1$. System $G_{1}(s)$ is a typical second kind elementary fractional-order system which has been frequently used in control performance tests [28], and system $G_{2}(s)$ is the most widely applied first kind elementary fractional-order system [29]. Parameters of the proposed controller are achieved as $k=29.3, k_{p}=14.65, k_{i}=29.3$, $k_{d}=23.44, \lambda=0.9$, and $\mu=1.3$ for system $G_{1}(s)$, and $k=$ 17.6, $k_{i}=17.6, k_{d}=17.6, \lambda=1.03$, and $\mu=0.47$ for system $G_{2}(s)$. For comparisons, another two controllers are used to control the same systems, namely, the optimal fractionalorder PID controller (FOPID) proposed in [21] and the PID controller used in [4]. The controlled systems should be robust to different problems, such as parameter uncertainties, noise, and disturbance. Therefore, the robustness test in this section will be demonstrated in these aspects, namely, set-point tracking performance with gain uncertainties, noise suppression, and disturbance rejection.

5.1. Set-Point Tracking with Gain Uncertainties. The unit step input tracking performances of systems $G_{1}(s)$ and $G_{2}(s)$ controlled by three controllers (the proposed controller is named as Robust FOPID) are shown in Figures 8 and 9. It can be seen that, for both systems, the control performance of Robust FOPID controller outperforms the other two controllers with no overshoot, shorter settling time, and rising time. The control performance of FOPID is also acceptable with relatively small overshoot, reasonable settling time, and rising time. However, the overshoot of $G_{1}(s)$ and the settling time of both $G_{1}(s)$ and $G_{2}(s)$ are quite large with PID controller.

Then, the set-point tracking performances of systems $G_{1}(s)$ and $G_{2}(s)$ with $\pm 30 \%$ gain uncertainties are illustrated in Figures 10 and 11. The shadowed spaces in different 


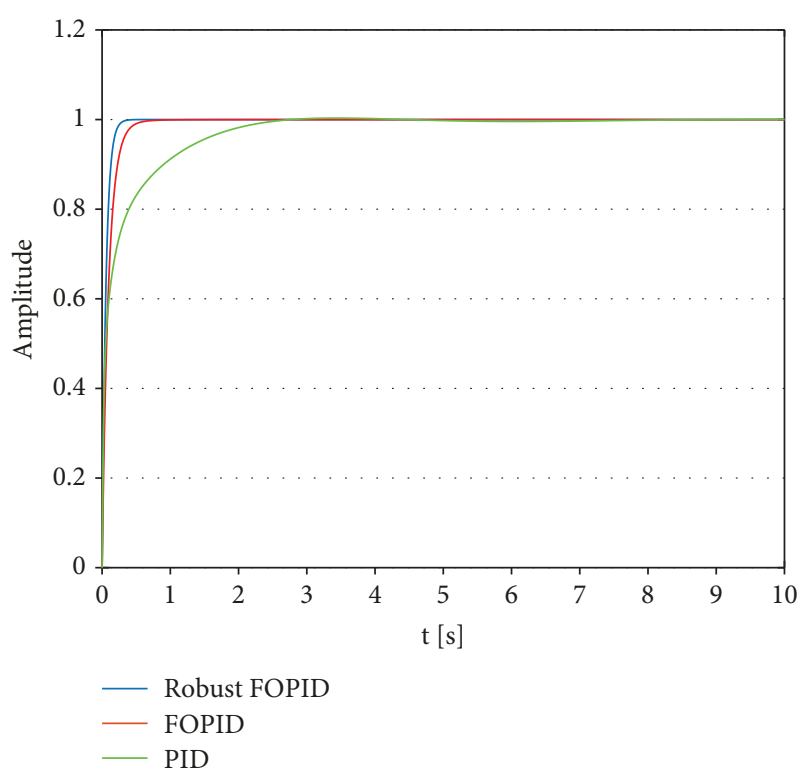

Figure 9: Step response comparison of $G_{2}(s)$.

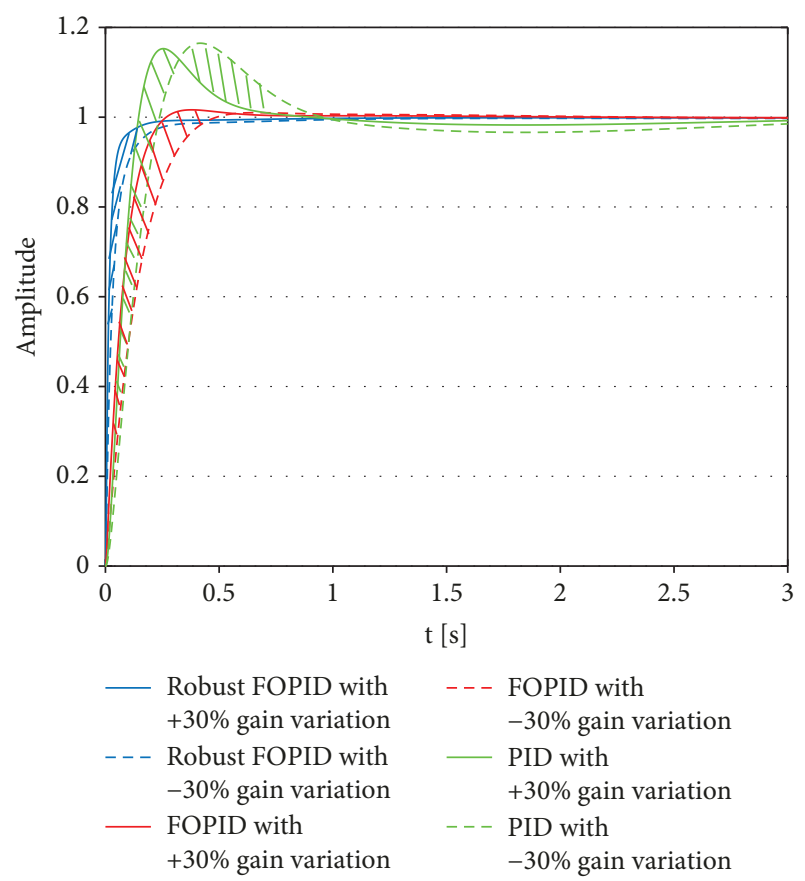

Figure 10: Step response comparison of $G_{1}(s)$ with gain uncertainties.

colours show the performance change gaps of systems with $\pm 30 \%$ gain uncertainties. For both $G_{1}(s)$ and $G_{2}(s)$, the performance gap with the proposed Robust FOPID controller is quite narrow, which means that the system is fairly robust to gain variations. The width of the performance gap of systems controlled by FOPID controller is wider than that of Robust FOPID controller, and the corresponding overshoots and rising times change moderately with gain variation. The largest performance gap appears in the system

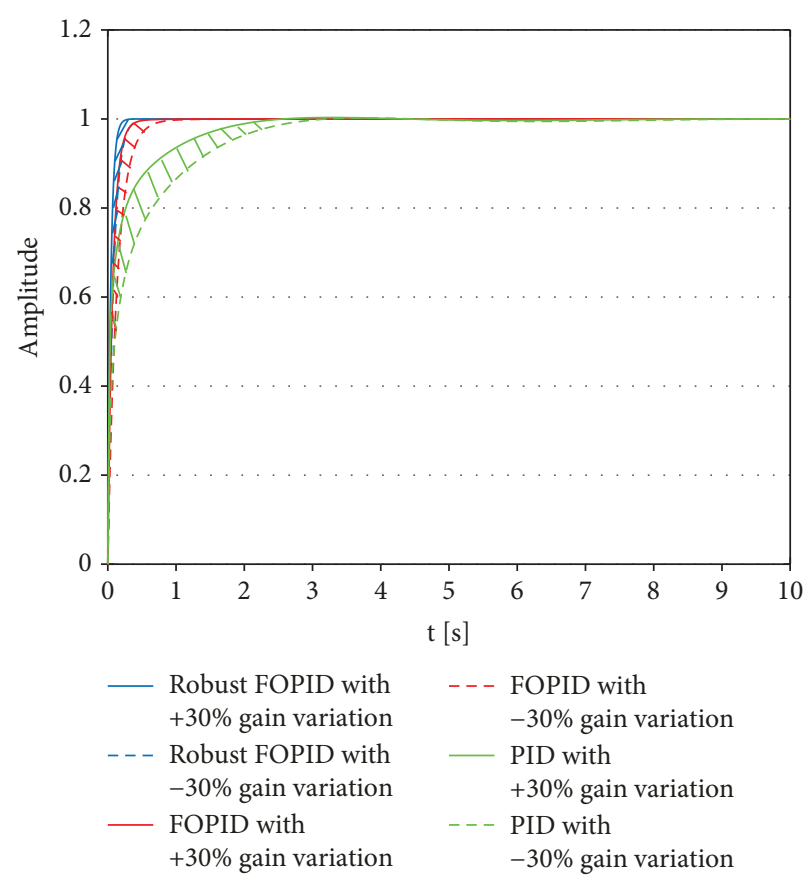

FIgURE 11: Step response comparison of $G_{2}(s)$ with gain uncertainties.

performances controlled by PID controller. Therefore, the robustness to parameter uncertainties of the systems controlled by the proposed Robust FOPID controller outperforms the other two.

5.2. Noise Suppression. Robustness of the controlled systems with respect to noise suppression is tested in this subsection. A random noise with amplitude -0.03 to +0.03 and sampling time 0.01 and another random noise with amplitude -0.05 to +0.05 and sampling time 0.005 are added into the feedback path of systems $G_{1}(s)$ and $G_{2}(s)$, respectively. The effects of the noise inputs are demonstrated in Figures 12 and 13. From the comparisons, it is shown that the control performance of Robust FOPID controller is better than those of the other two with relatively smaller fluctuation. Hence, the noise suppression ability of the proposed robust FOPID controller is more superior to the other ones.

5.3. Disturbance Rejection. Another vital concern for control system is their disturbance rejection ability. Therefore, in this subsection, an external disturbance with amplitude -0.2 which lasts for 0.05 seconds and another one with amplitude -0.25 which also lasts for 0.05 seconds are added to $G_{1}(s)$ and $G_{2}(s)$ at $t=3 \mathrm{~s}$, respectively. Figures 14 and 15 show the disturbance rejection performances of the two systems. It is illustrated that systems controlled by Robust FOPID controller return back to the set-point value quickly after the appearance of external disturbance. However, the corresponding recovering times are quite long for the systems controlled by FOPID and PID controllers.

On the whole, the robust control performances of different controlled systems with the proposed controller 


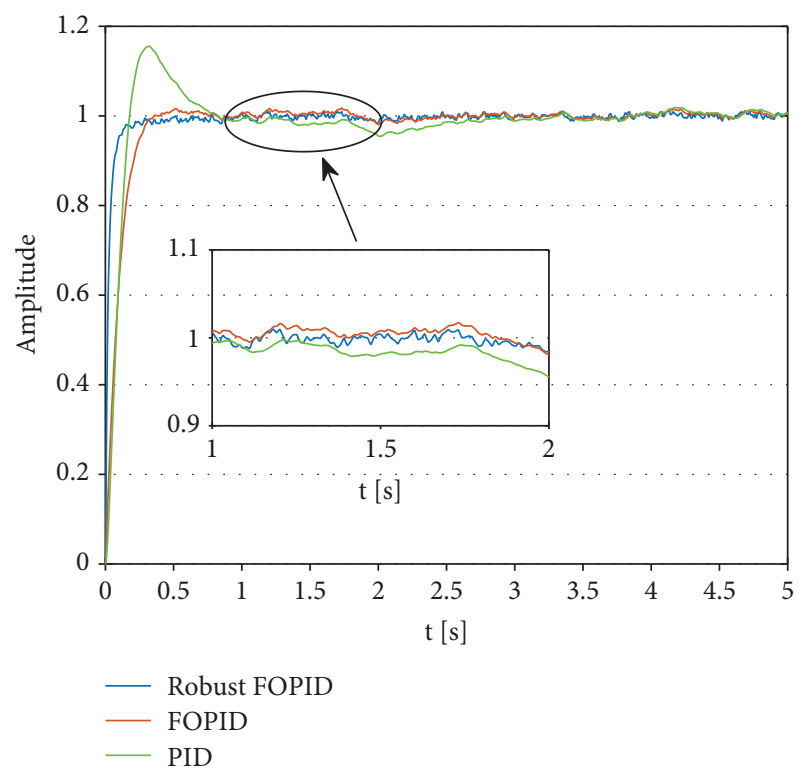

FIGURE 12: Step response comparison of $G_{1}(s)$ with noise input.

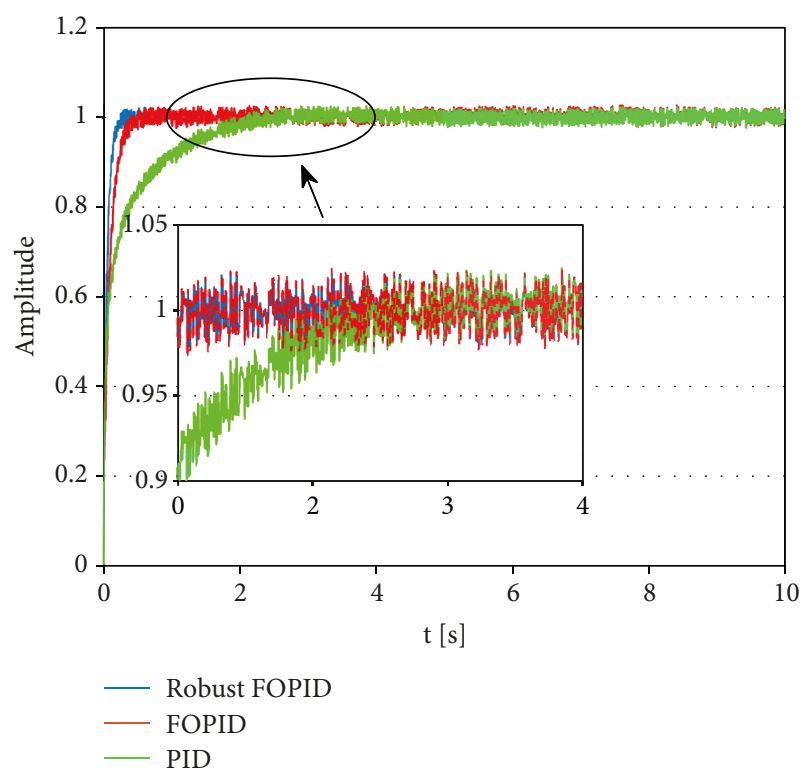

FIGURE 13: Step response comparison of $G_{2}(s)$ with noise input.

are quite satisfactory, which outperform the other controllers in set-point tracking with parameter uncertainties, noise suppression, and disturbance rejection aspects.

\section{Conclusion}

A robust FOPID controller regulation algorithm based on small gain theorem is studied in this paper. The robustness analysis is achieved by the constructed robustness evaluation function. Two control parameters $k$ and $\beta$ are discussed to help the controlled system obtain better robust performance, but only $k$ is necessary to be tuned. In

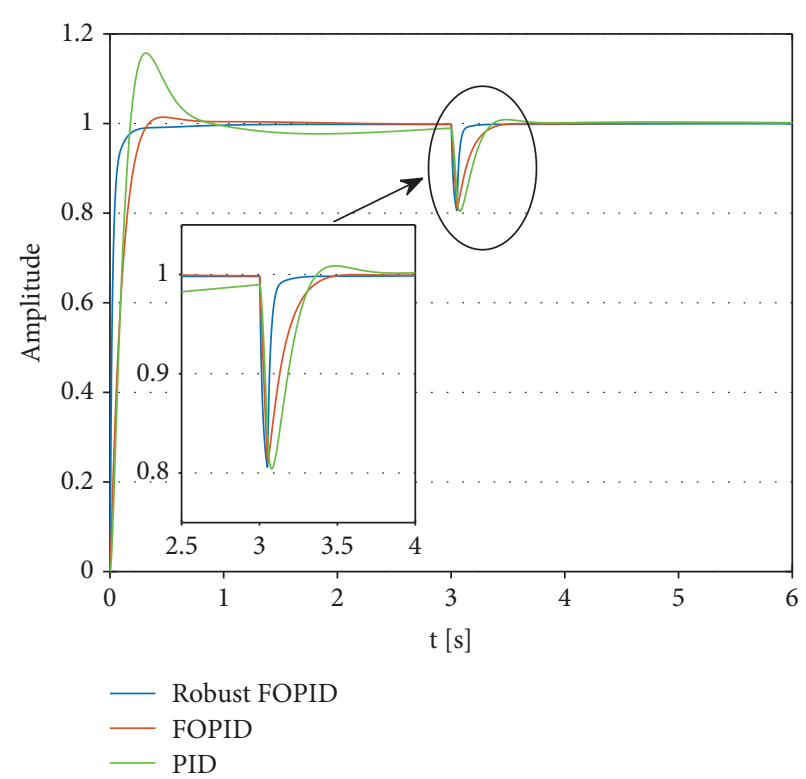

FIGURE 14: Step response comparison of $G_{1}(s)$ with disturbance.

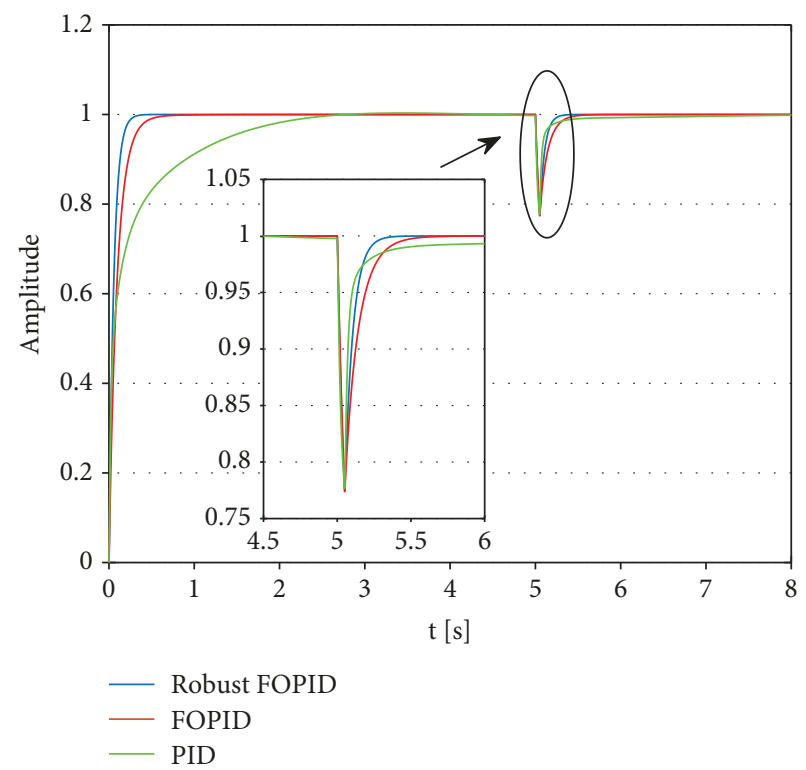

FIGURE 15: Step response comparison of $G_{2}(s)$ with disturbance.

addition, some robustness discussion of FOPI and FOID controllers for a specific kind of frequently used fractionalorder system is also demonstrated. The proposed controller is simple and easy to be applied on both fractional-order and integer-order systems. Simulation examples of system control performance under different conditions, namely, with parameter uncertainties, external disturbance, and noise, are illustrated to show the effectiveness of the proposed regulation algorithm. In our next work, we may concentrate on time delay problem, namely, the relationship between the robustness of controlled system and time delay using the proposed evaluation function. 


\section{Data Availability}

The data used to support the findings of this study are available from the corresponding author upon request.

\section{Conflicts of Interest}

The authors declare that there is no conflict of interest regarding the publication of this paper.

\section{Acknowledgments}

This work is partially supported by the National Natural Science Foundation of China under Grant no. 61673094 and the Fundamental Research Funds for the Central Universities of China under Grant nos. G2018KY0305 and G2018KY0302.

\section{References}

[1] H. Sun, W. Chen, and Y. Chen, "Variable-order fractional differential operators in anomalous diffusion modeling," Physica A: Statistical Mechanics and its Applications, vol. 388, no. 21, pp. 4586-4592, 2009.

[2] R. L. Magin, "Fractional calculus models of complex dynamics in biological tissues," Computers \& Mathematics with Applications, vol. 59, no. 5, pp. 1586-1593, 2010.

[3] Y. Chen, I. Petras, and D. Xue, "Fractional order control - a tutorial," in 2009 American Control Conference, pp. 13971411, St. Louis, MO, USA, June 2009.

[4] L. Liu, F. Pan, and D. Xue, "Variable-order fuzzy fractional PID controller," ISA Transactions, vol. 55, pp. 227-233, 2015.

[5] I. Podlubny, "Fractional-order systems and $\mathrm{PI}^{\lambda} \mathrm{D}^{\mu}$-controllers," IEEE Transactions on Automatic Control, vol. 44, no. 1, pp. 208-214, 1999.

[6] S. Zhang, Y. Yu, and H. Wang, "Mittag-Leffler stability of fractional-order Hopfield neural networks," Nonlinear Analysis: Hybrid Systems, vol. 16, pp. 104-121, 2015.

[7] S. Zhang, Y. Yu, and Q. Wang, "Stability analysis of fractionalorder Hopfield neural networks with discontinuous activation functions," Neurocomputing, vol. 171, pp. 1075-1084, 2016.

[8] Y. Arya, "AGC of two-area electric power systems using optimized fuzzy PID with filter plus double integral controller," Journal of the Franklin Institute, vol. 355, no. 11, pp. 45834617, 2018.

[9] Y. Luo and Y. Chen, Fractional Order Motion Controls, John Wiley \& Sons Ltd., Chichester, UK, 2013.

[10] J. Chen, B. Zhuang, Y. Chen, and B. Cui, "Diffusion control for a tempered anomalous diffusion system using fractional-order PI controllers," ISA Transactions, 2017.

[11] L. Liu, S. Tian, D. Xue, T. Zhang, and Y. Chen, "Continuous fractional-order zero phase error tracking control," ISA Transactions, vol. 75, pp. 226-235, 2018.

[12] Y. Wei, Y. Chen, S. Cheng, and Y. Wang, "Completeness on the stability criterion of fractional order LTI systems," Fractional Calculus and Applied Analysis, vol. 20, no. 1, pp. 159-172, 2017.

[13] T. J. Freeborn, "A survey of fractional-order circuit models for biology and biomedicine," IEEE Journal on Emerging and Selected Topics in Circuits and Systems, vol. 3, no. 3, pp. 416424, 2013.
[14] I. Petras, "Fractional-order feedback control of a DC motor," Journal of Electrical Engineering, vol. 60, pp. 117-128, 2009.

[15] Y. Luo and Y. Chen, "Fractional order [proportional derivative] controller for a class of fractional order systems," Automatica, vol. 45, no. 10, pp. 2446-2450, 2009.

[16] J. Chen, B. Zhuang, Y. Chen, and B. Cui, "Backstepping-based boundary feedback control for a fractional reaction diffusion system with mixed or Robin boundary conditions," IET Control Theory \& Applications, vol. 11, no. 17, pp. 29642976, 2017.

[17] B. M. Vinagre, I. Podlubny, L. Dorcak, and V. Feliu, "On fractional PID controllers: a frequency domain approach," in Proceedings of IFAC Workshop on Digital Control: Past, Present and Future of PID Control, pp. 53-58, Terrasa, Spain, 2000.

[18] C. A. Monje, B. M. Vinagre, V. Feliu, and Y. Chen, "Tuning and auto-tuning of fractional order controllers for industry applications," Control Engineering Practice, vol. 16, no. 7, pp. 798-812, 2008.

[19] S. E. Hamamci, "An algorithm for stabilization of fractionalorder time delay systems using fractional-order PID controllers," IEEE Transactions on Automatic Control, vol. 52, no. 10, pp. 1964-1969, 2007.

[20] M. Zamani, M. Karimi Ghartemani, N. Sadati, and M. Parniani, "Design of a fractional order PID controller for an AVR using particle swarm optimization," Control Engineering Practice, vol. 17, no. 12, pp. 1380-1387, 2009.

[21] D. Xue, Fractional-Order Control Systems: Fundamentals and Numerical Implementations, de Gruyter, Berlin, 2017.

[22] W. Deng, C. Li, and J. Lu, "Stability analysis of linear fractional differential system with multiple time delays," Nonlinear Dynamics, vol. 48, no. 4, pp. 409-416, 2007.

[23] D. Matignon, "Stability results for fractional differential equations with applications to control processing," Computational Engineering in Systems Applications, vol. 2, pp. 963-968, 1996.

[24] R. Azarmi, M. Tavakoli-Kakhki, A. K. Sedigh, and A. Fatehi, "Analytical design of fractional order PID controllers based on the fractional set-point weighted structure: case study in twin rotor helicopter," Mechatronics, vol. 31, pp. 222233, 2015.

[25] H. Ozbayabb, "PID controller design for fractional-order systems with time delays," Systems \& Control Letters, vol. 61, no. 1, pp. 18-23, 2012.

[26] F. Padula, R. Vilanova, and A. Visioli, " $H_{\infty}$ optimizationbased fractional-order PID controllers design," International Journal of Robust and Nonlinear Control, vol. 24, no. 17, pp. 3009-3026, 2013.

[27] D. Wang and X. Gao, " $H_{\infty}$ design with fractional-order $P D^{\mu}$ controllers,” Automatica, vol. 48, pp. 974-977, 2012.

[28] L. Liu and S. Zhang, "Robust fractional-order PID controller tuning based on Bode's optimal loop shaping," Complexity, vol. 2018, Article ID 6570560, 14 pages, 2018.

[29] R. Malti, X. Moreau, F. Khemane, and A. Oustaloup, "Stability and resonance conditions of elementary fractional transfer functions," Automatica, vol. 47, no. 11, pp. 2462-2467, 2011. 


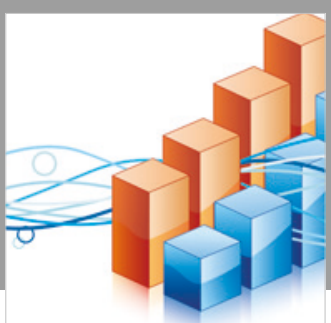

Advances in

Operations Research

\section{-n-m}
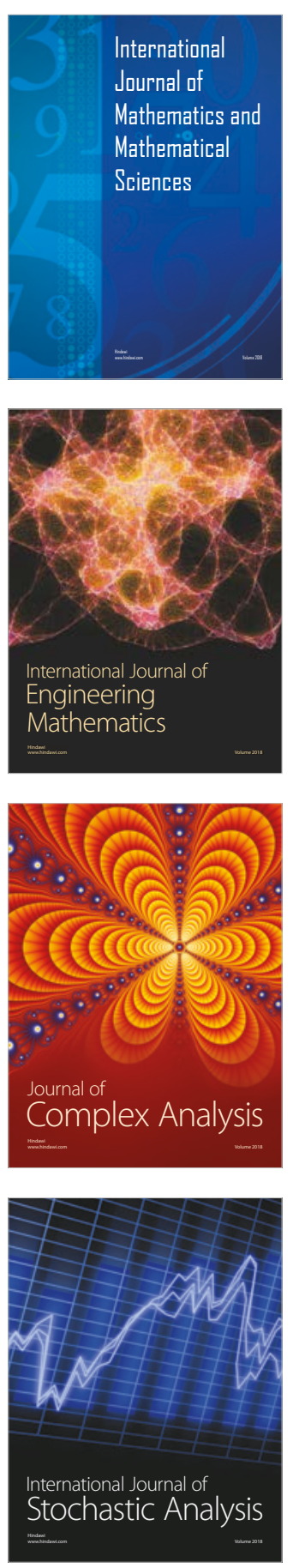
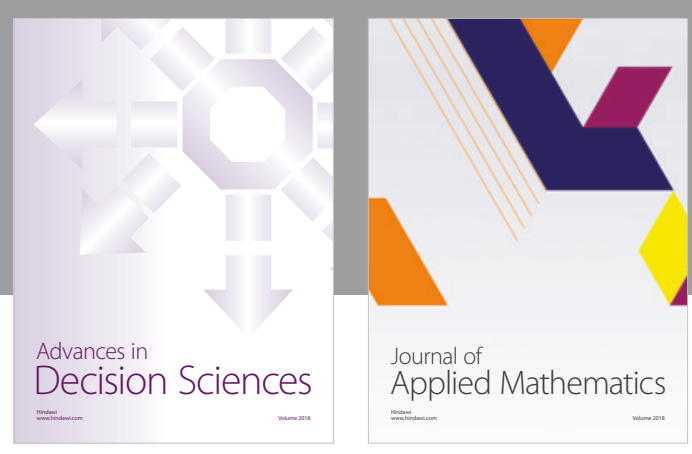

Journal of

Applied Mathematics
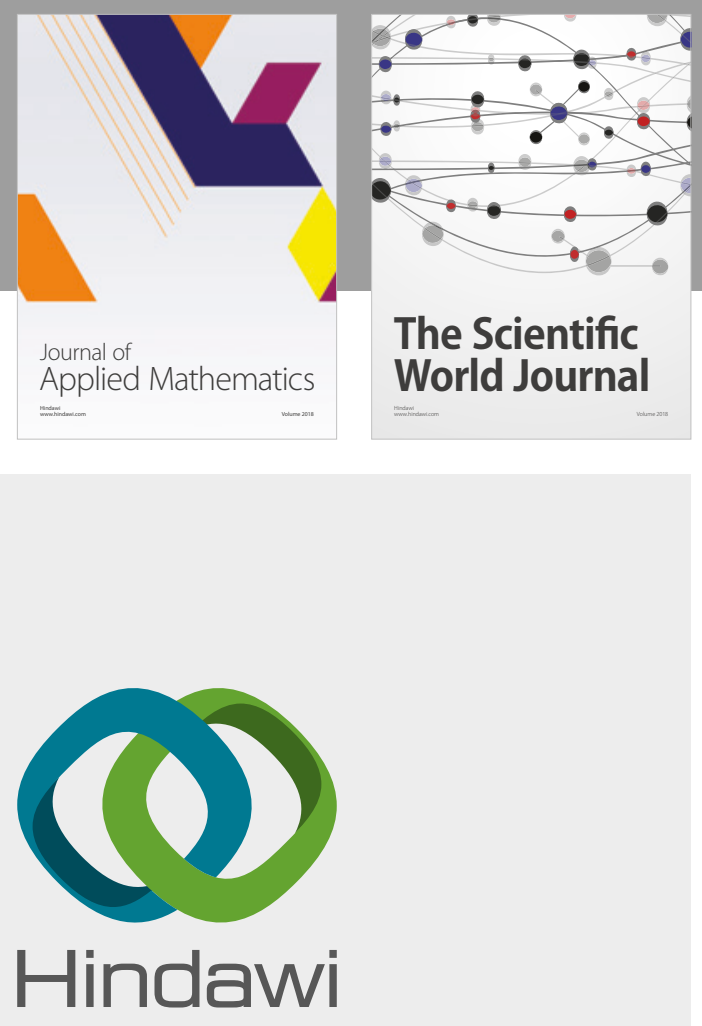

Submit your manuscripts at

www.hindawi.com

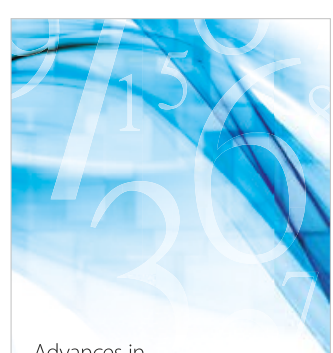

Advances in
Numerical Analysis
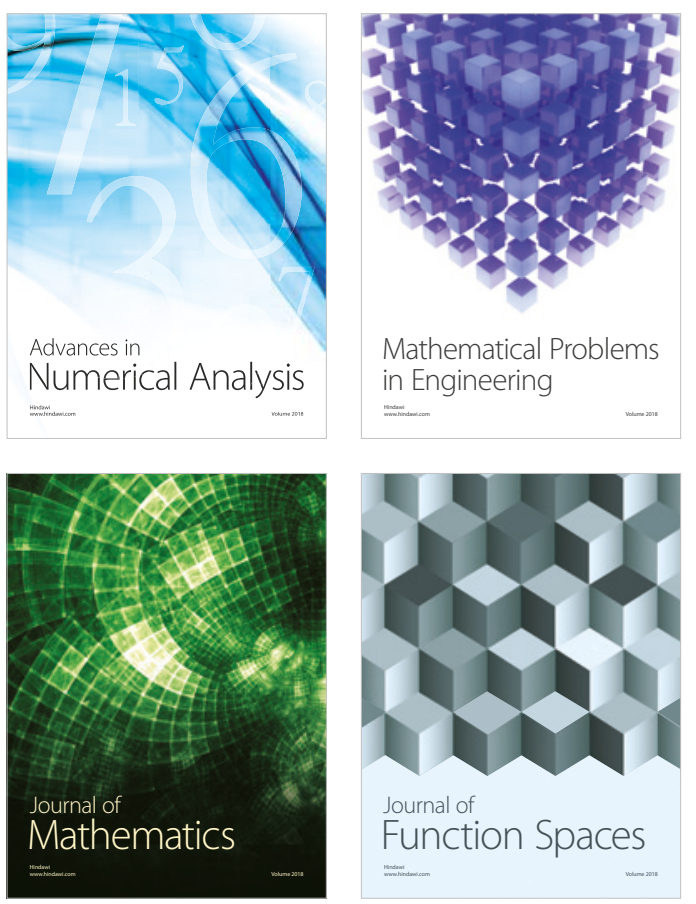

Mathematical Problems in Engineering

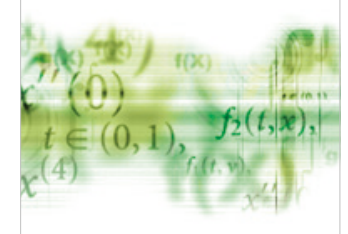

International Journal of

Differential Equations

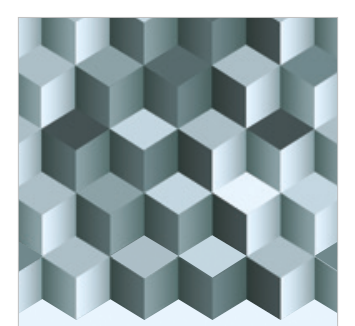

Journal of

Function Spaces
The Scientific

World Journal

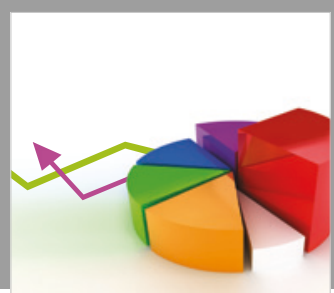

Journal of

Probability and Statistics
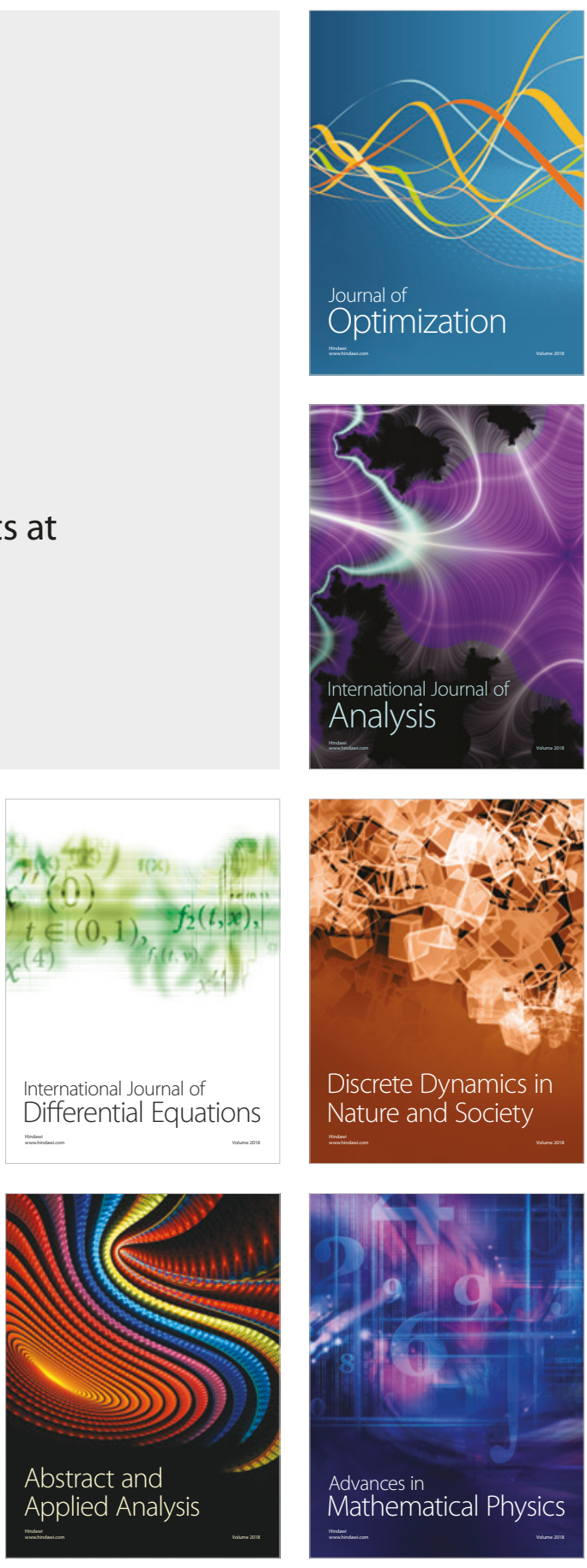